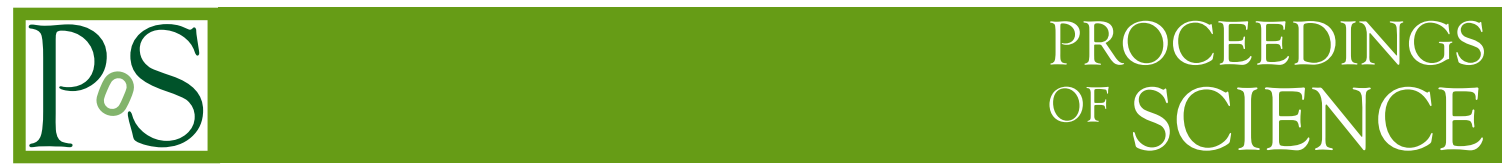

\title{
Higgs pair production in the Composite Higgs model
}

\author{
Ramona Gröber* \\ Institut für Theoretische Physik, Karlsruhe Institute of Technology, 76128 Karlsruhe, Germany \\ E-mail: ramona@particle.uni-karlsruhe.de
}

\section{Margarete Mühlleitner}

Institut für Theoretische Physik, Karlsruhe Institute of Technology, 76128 Karlsruhe, Germany

E-mail: maggie@particle.uni-karlsruhe.de

In composite Higgs models the Higgs boson arises as a pseudo Nambu-Goldstone boson of an enlarged global symmetry. The Higgs potential is generated by loops of Standard Model fermions and gauge bosons. To reconstruct the Higgs potential trilinear and quartic couplings must be measured. In the composite Higgs model these couplings are modified compared to the SM and they depend on a new parameter - the compositeness parameter $\xi$. In this talk the Higgs pair production cross sections in two composite Higgs models will be represented. The prospects of extracting the triple Higgs coupling will be discussed.

Proceedings of the Corfu Summer Institute 2011 School and Workshops on Elementary Particle Physics and Gravity

September 4-18, 2011

Corfu, Greece

${ }^{*}$ Speaker. 


\section{Introduction}

The Standard Model (SM) of particle physics which has been very successful in describing the strong, weak and electromagnetic interactions is based on an $S U(3)_{C} \times S U(2)_{L} \times U(1)_{Y}$ gauge symmetry which is broken down to an $S U(3)_{C} \times U(1)_{e m}$. The mechanism of electroweak symmetry breaking is implemented by a complex scalar field acquiring a vacuum expectation value (VEV). This field transforms as a doublet under the $S U(2)$. Three of the four degrees of freedom of the $S U$ (2)-doublet become the longitudinal modes of the massive gauge bosons; one degree of freedom is left and can be associated with the Higgs boson [1-3]. The Higgs sector of the SM is highly restricted and the interactions of the Higgs boson to the fermions and gauge bosons are proportional to their masses. The only free parameter of the Higgs sector is the Higgs boson mass. The Higgs boson has another very important feature: It unitarizes the scattering of longitudinal gauge bosons and therefore plays the role of an UV-moderator.

However, the simple description of the electroweak symmetry breaking (EWSB) in the SM which only introduces a single fundamental scalar field has its problems. As the Higgs boson mass is not protected by any symmetry, the Higgs mass suffers from radiative instabilities. The Higgs mass is quadratically divergent in the cut-off scale $\Lambda$ up to which the SM is assumed to be valid. This makes fine-tuning necessary in order to ensure that the Higgs mass is of the order of the electroweak symmetry breaking scale.

In order to solve this problem several models beyond the SM have been discussed. One of the maybe most established examples is supersymmetry [4]. The SM particles get superpartners with spins that differ by a half from the corresponding SM partner. This leads to a cancellation of the quadratic divergences.

Another alternative is that the Higgs boson is not a fundamental scalar particle but a composite scalar arising as a pseudo Nambu- Goldstone boson from an enlarged global symmetry [5]. Therefore, the Higgs mass is protected by this symmetry from large radiative corrections. However, since the global symmetry is only approximate, loops of SM fermions and gauge bosons generate a Higgs potential nevertheless.

In such a class of models the Higgs to fermion couplings, the Higgs to gauge boson couplings and the Higgs self-couplings are modified. This can be tested at the LHC. Although such a deviation from the SM couplings is no direct probe of the strong sector it would give a first hint of the nature of electroweak symmetry breaking.

In order to understand the origin of electroweak symmetry breaking a precise measurement of the Higgs potential is necessary. To achieve this the Higgs self-couplings must be measured. The prospects of measuring the trilinear Higgs coupling in Minimal Composite Higgs models are discussed in the following. More details can be found in Ref. [6].

\section{Minimal Composite Higgs models}

For this talk we will concentrate on the Minimal Composite Higgs models of Refs. [7,8] which are based on a 5d description in Anti-de-Sitter space time. There exists an holographic description in terms of a $4 d$ strongly-coupled theory. In such an effective low-energy description the effects of the strongly-interacting sector can be described by a single new parameter $\xi$ which is defined as 


\begin{tabular}{|c|c|c|c|c|c|}
\hline & $H V V$ & $H H V V$ & $H H H$ & $H H H H$ & $H f \bar{f}$ \\
\hline $\mathrm{MCHM}_{4}$ & $\sqrt{1-\xi}$ & $1-2 \xi$ & $\sqrt{1-\xi}$ & $\left(1-\frac{7}{3} \xi\right)$ & $\sqrt{1-\xi}$ \\
\hline $\mathrm{MCHM}_{5}$ & $\sqrt{1-\xi}$ & $1-2 \xi$ & $\frac{1-2 \xi}{\sqrt{1-\xi}}$ & $\frac{1-\frac{28}{3}(1-\xi) \xi}{1-\xi}$ & $\frac{1-2 \xi}{\sqrt{1-\xi}}$ \\
\hline
\end{tabular}

Table 1: Modification factors of the $\mathrm{MCHM}_{4}$ and $\mathrm{MCHM}_{5}$ with respect to the $\mathrm{SM}$ for the Higgs to vector boson couplings ( $H V V$ and $H H V V$ ) the Higgs self-couplings ( $H H H$ and $H H H H$ ) and the coupling of one Higgs boson to fermions $(H f \bar{f})$.

$\xi=\frac{v^{2}}{f^{2}}$ where $v=246 \mathrm{GeV}$ is the electroweak symmetry breaking scale and $f$ is the scale where the breaking of the global symmetry occurs. The value $\xi=0$ corresponds to the SM and $\xi=1$ is the technicolor limit. In the Minimal Composite Higgs models the bulk symmetry $S O(5) \times U(1)$ is broken at a scale $f$ to a subgroup $S O(4) \times U(1)$ on the IR boundary. On the UV brane the global symmetry is broken down to the SM gauge group. In the model of Ref. [7] $\left(\mathrm{MCHM}_{4}\right)$ the fermions are in the spinorial representation. They transform in the fundamental representation in the $\mathrm{MCHM}_{5}$ (model of Ref. [8]). ${ }^{1}$

The Higgs self-couplings and the Higgs fermion couplings depend on the representation of the SM fermions whereas the Higgs gauge boson couplings are independent of it. In Table 1 the modification factors of these couplings can be found.

For $\xi=0.5$ in the $\mathrm{MCHM}_{5}$ the couplings of the Higgs boson to fermions of the SM vanish as well as the triple Higgs coupling. This means that we have a fermiophobic scenario for $\xi=0.5{ }^{2}$ In both models the two Higgs bosons to two vector boson couplings vanish for $\xi=0.5$.

A new coupling which plays an important role for Higgs pair production is the coupling of two Higgs bosons to two fermions. It is given by

$$
\begin{array}{lll}
\mathrm{MCHM}_{4}: & H H f \bar{f}: & \xi \frac{m_{f}}{v^{2}} \\
\mathrm{MCHM}_{5}: & H H f \bar{f}: & 4 \xi \frac{m_{f}}{v^{2}}
\end{array}
$$

Note that this coupling vanishes in the SM limit $\xi \rightarrow 0$ as expected.

The new parameter $\xi$ as well as the Higgs mass is restricted by direct searches as well as by electroweak precision data. Due to the LEP data, masses lower than $114 \mathrm{GeV}$ can be excluded for most values of $\xi$ but the exclusion bounds can be relaxed a bit for $\xi$ in the region around 0.5 . Tevatron excludes the mass region between $\sim 157-175 \mathrm{GeV}$ for low values of $\xi$. For the $\mathrm{MCHM}_{5}$ an additional region for large values of $\xi$ between $150-200 \mathrm{GeV}$ is excluded. More details on this can be found in Ref. [10]. Direct searches from ATLAS and CMS [11] mainly restrict the low $\xi$ range similar to the $\mathrm{SM}$ constraints. For the $\mathrm{MCHM}_{5}$, some additional areas for high values of $\xi$ are also excluded by LHC searches. Plots including the results from December 2011 can be found

\footnotetext{
${ }^{1}$ In the $\mathrm{MCHM}_{5}$ the subgroup to which the bulk symmetry is broken is enlarged to an $O(4) \times U(1)$. This relaxes constraints from the $Z b_{L} \bar{b}_{L}$ coupling.

${ }^{2}$ Despite the vanishing couplings the fermions still get a mass since the direct coupling to the vacuum expectation value is non-zero. This can be inferred from the Lagrangian given in Ref. [9]. There are new kinetic terms for the Higgs fields. In order to absorb them the Higgs field must be redefined. This means that there is a non-linear dependence on the Higgs field in the Higgs doublet. This and additional six dimensional operators lead to a redefinition of the VEV and the Higgs, gauge boson and fermion masses.
} 
in [12]. More constraints come from the $S$ and $T$ parameters. They impose that $\xi \lesssim 0.3$. But this bound can be relaxed if one assumes that there is a partial cancellation of the order of $50 \%$ with contributions from other states.

\section{Higgs pair production at the LHC}

The measurement of Higgs self-couplings is an difficult but important task since it enables the reconstruction of the Higgs potential and hence gives an insight into the mechanism of electroweak symmetry breaking. For the SM it was found that the quartic Higgs coupling cannot be measured at either a high-luminosity LHC $[13,14]$ nor a high-energy linear collider $[14,15]$. So in the following we will concentrate on the measurement of the triple Higgs coupling which is accessible in Higgs pair production.

At the LHC the most important process in the SM is Higgs pair production via gluon fusion [16] followed by $W W / Z Z$ fusion [17] and double Higgs-strahlung [18]. The corresponding Feynman diagrams can be found in Fig. 1. Note that for the gluon fusion process there is a new diagram originating from the new coupling of two Higgs bosons to two fermions.

$\underline{g g \text { double Higgs fusion: } g g \rightarrow H H}$
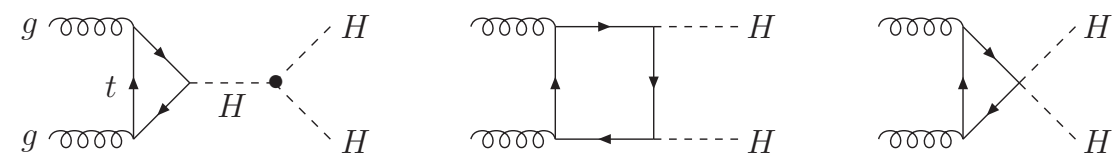

$\underline{W W / Z Z \text { double Higgs fusion: } q q \rightarrow q q H H}$
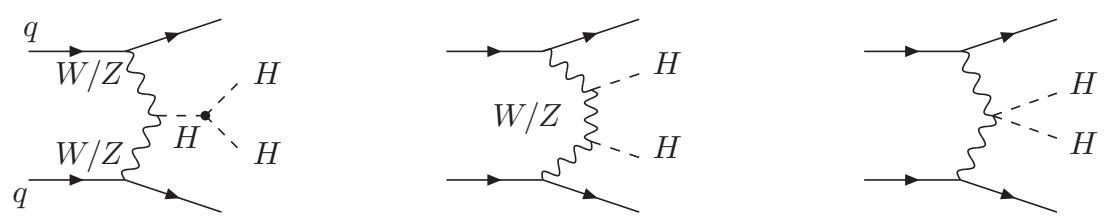

double Higgs-strahlung: $q \bar{q} \rightarrow Z H H / W H H$
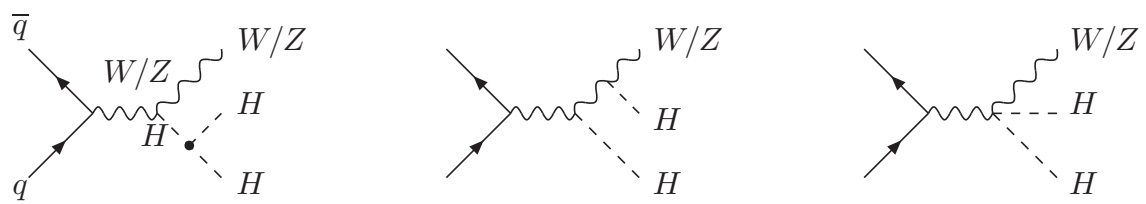

Figure 1: Generic diagrams of the Higgs pair production processes at the LHC in the composite Higgs model: $g g$ fusion, $W W / Z Z$ fusion and double Higgs-strahlung.

In Fig. 2 the cross sections for the three processes for the $\mathrm{MCHM}_{4}$ as a function of the Higgs boson mass for the SM and three characteristic values of $\xi$ can be found. The arrows in the plots 
indicate a change in the triple Higgs coupling from 50\% to $150 \%$ and are hence an indicator for the sensitivity on the triple Higgs coupling. In Fig. 3 the corresponding cross sections for the $\mathrm{MCHM}_{5}$ are shown. For the gluon fusion process we included the QCD corrections by multiplying with a $\mathrm{K}$-factor of $\sim 2$ [19]. For the $\mathrm{MCHM}_{4}$, the cross section for the gluon fusion process is enhanced for all values of $\xi$ compared to the SM. This is due to the diagram with the new coupling. It is not suppressed by an extra propagator as it is the case for the diagram involving the triple Higgs coupling. Therefore, it plays an important role especially for large values of $\xi$ where the coupling strength increases. This implies that the sensitivity to the triple Higgs coupling becomes smaller not only because the coupling itself becomes smaller with increasing $\xi$ but also because the diagram involving the triple Higgs coupling is overwhelmed by contribution from the new diagram. Also the cross section for vector boson fusion ${ }^{3}$ increases which is mainly due to interference effects. In double Higgs-strahlung the cross section in the $\mathrm{MCHM}_{4}$ is always smaller than in the SM due to decreasing coupling strength and destructive interference.

In the $\mathrm{MCHM}_{5}$, the cross section for gluon fusion is even more enhanced with $\xi$ than in the $\mathrm{MCHM}_{4}$. On the one side this is due to the coupling $H H f \bar{f}$ which is a factor of 4 larger. On the other hand also the other couplings are larger for large values of $\xi$ than in the $\mathrm{MCHM}_{4}$. Note that there is no sensitivity at all on the triple Higgs coupling for all three processes for $\xi=0.5$ because the triple Higgs coupling vanishes for this value of $\xi$.

In order to investigate the expected experimental sensitivity to a deviation in the triple Higgs coupling the decays of the Higgs bosons must be taken into account. We adopted the narrowwidth-approximation where the production cross section is multiplied by the branching ratios. In Fig. 4 the branching ratios for the $\mathrm{MCHM}_{4}$ are displayed. Since in $\mathrm{MCHM}_{4}$ all couplings are changed by the same factor compared to the SM the branching ratios are the same as in the SM. As can be inferred from the figure, the Higgs boson dominantly decays into $b \bar{b}$ until the gauge boson threshold is reached. Above $M_{H} \sim 140 \mathrm{GeV}$ the dominant decay channel is $H \rightarrow W^{+} W^{-}$followed by $H \rightarrow Z Z$.

In the $\mathrm{MCHM}_{5}$ the branching ratios depend on $\xi$. In Fig. 5 the branching ratios are given for two typical Higgs boson masses as a function of $\xi$. For low Higgs boson masses the decays into $b \bar{b}$ dominate. For $\xi=0.5$ the fermionic branching ratios become zero, since the coupling of the Higgs boson to the fermions vanishes. Also the branching ratio of the decay into two gluons mediated by fermion loops becomes zero in this case. Therefore in the region around $\xi=0.5$ the Higgs boson decays mainly into $W^{+} W^{-}$. Also the decay into $\gamma \gamma$ is enhanced which leads to a rather clear signal in the Higgs boson searches. For a Higgs mass of $M_{H}=180 \mathrm{GeV}$ the decays into $W^{+} W^{-}$dominate followed by the ones into $Z Z$. For very large values of $\xi$, however, the fermionic branching ratios become more important because the couplings to vector bosons become smaller and the couplings to fermions larger.

In order to investigate whether the triple Higgs coupling can be measured at the LHC we concentrate on the dominating process given by gluon fusion. In the following it is assumed that EWSB

\footnotetext{
${ }^{3}$ Higgs pair production via vector boson fusion is quite an important process since it is a direct probe of the strong interaction due to its special high-energy behaviour. As it was pointed out by Refs. [9,20] due to the modified couplings the amplitude $W_{L} W_{L} \rightarrow H H$ increases $\sim \xi_{s}$ where $s$ is the center-of-mass energy squared. This means that the composite Higgs boson cannot fully unitarize this process anymore due to its modified couplings compared to the SM Higgs boson. Partial wave unitarity, however, can be restored by other new resonances of the strongly-interacting sector [21].
} 

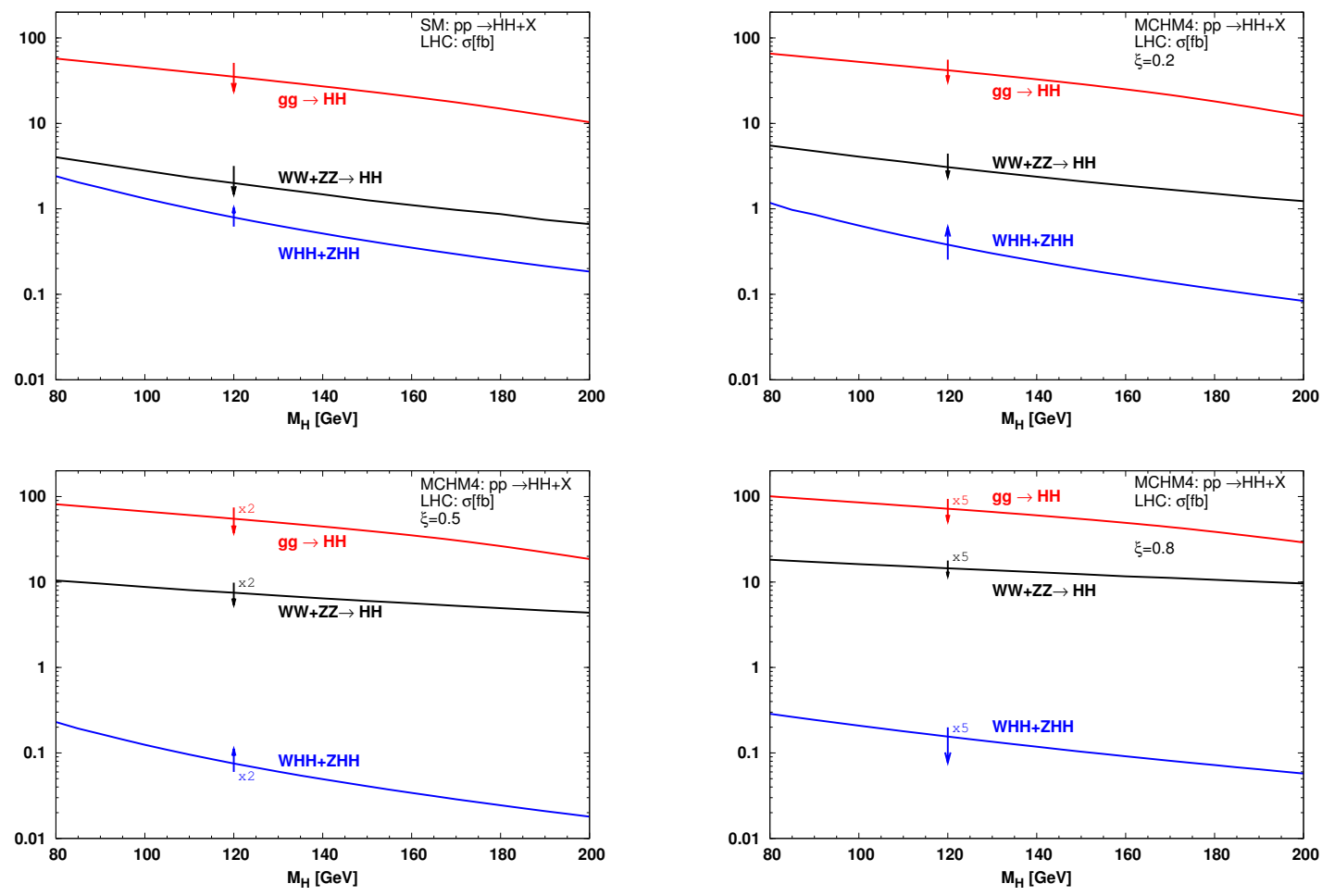

Figure 2: Higgs pair production cross sections as a function of the Higgs boson mass in the SM $(\xi=0$, upper left) and $\mathrm{MCHM}_{4}$ with $\xi=0.2$ (upper right), 0.5 (bottom left) and 0.8 (bottom right). Arrows indicate the change in the cross section for a variation of $\lambda_{H H H}$ from 0.5 to 1.5 times its value in the corresponding model. Some arrows are rescaled as indicated by appropriate factors to make them visible.

is realized in the framework of a Minimal Composite Higgs model and that all other couplings have been measured. ${ }^{4}$ For Higgs mass values above the gauge boson threshold the Higgs boson decaying into $W^{+} W^{-} W^{+} W^{-}$is the most interesting decay channel. For lower Higgs masses the decays into $4 b$-quarks give the largest number of signal events but the signal is completely overwhelmed by the large QCD background. Better prospects for a measurement of the triple Higgs coupling are given by the decay channels into $b \bar{b} \tau^{+} \tau^{-}$and $b \bar{b} \gamma \gamma$. For each Higgs mass we have determined the value of $\xi$ where the Higgs pair production cross section with subsequent decay in the different final states deviates by more than $1,2,3$ or $5 \sigma$ from the corresponding process where the triple Higgs coupling is set to zero. Denoting by $S$ the number of signal events for the process calculated in the composite Higgs model and by $S_{\lambda_{H H H}}$ the corresponding number where the triple Higgs coupling is set to zero, there is sensitivity to a non-vanishing triple Higgs coupling if

$$
S_{\lambda_{H H H}=0}>S+a \sqrt{S} \quad \text { or } \quad S_{\lambda_{H H H}=0}<S-a \sqrt{S} \quad \text { with } a=1,2,3,5 .
$$

For the $\mathrm{MCHM}_{4}$ the sensitivity areas can be found in Fig. 6. For the $b \bar{b} \gamma \gamma$ final state a measurement of the triple Higgs coupling with $5 \sigma$ is hopeless whereas for the $b \bar{b} \tau^{+} \tau^{-}$final state for the low Higgs mass range and the $W^{+} W^{-} W^{+} W^{-}$final state for large Higgs masses the prospects look much better. For the $\mathrm{MCHM}_{5}$ the corresponding plots can be found in Fig. 7. Around the region

\footnotetext{
${ }^{4}$ The value of $\xi$ can be measured with $20 \%$ precision [22].
} 

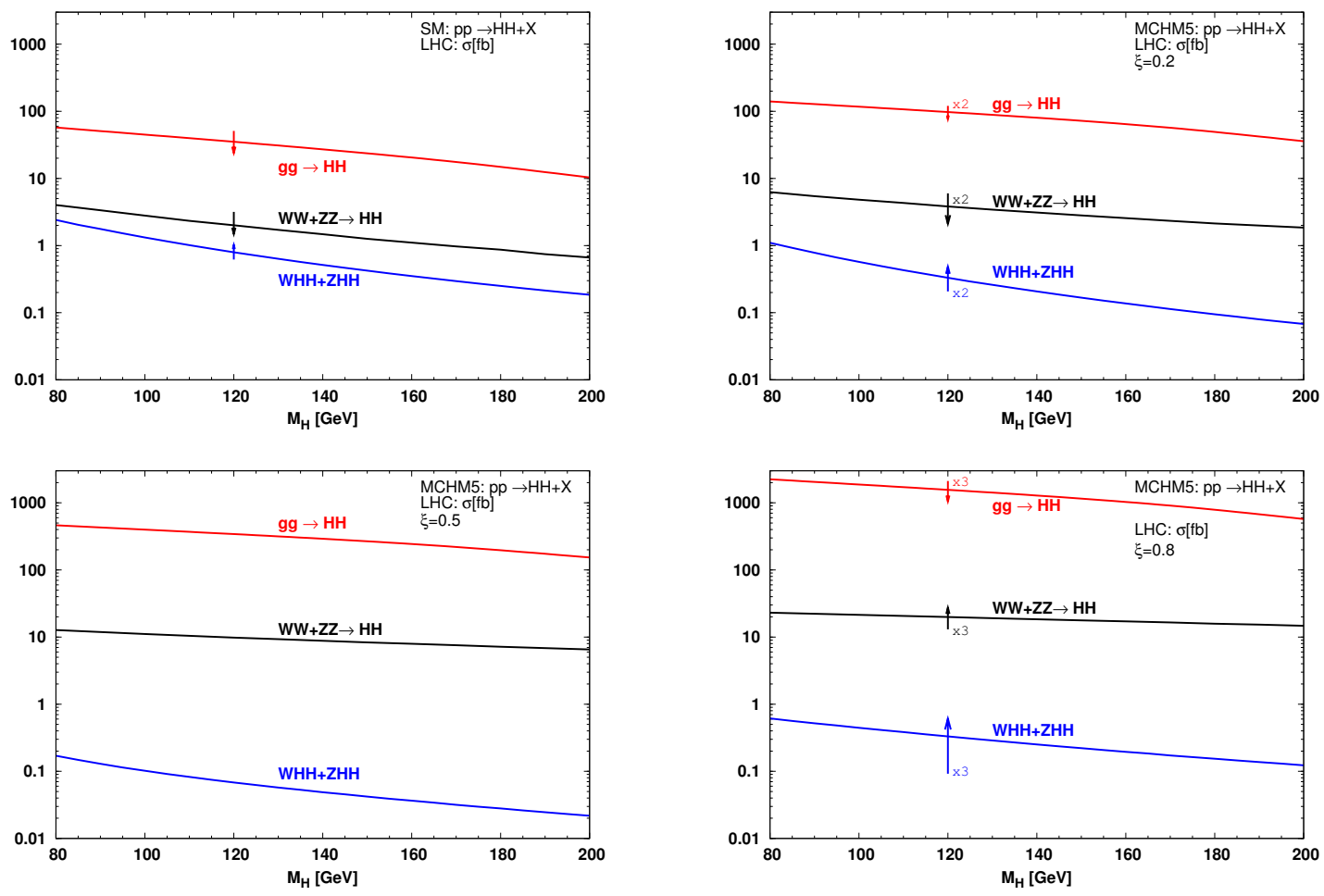

Figure 3: As in Fig. 2 but for $\mathrm{MCHM}_{5}$

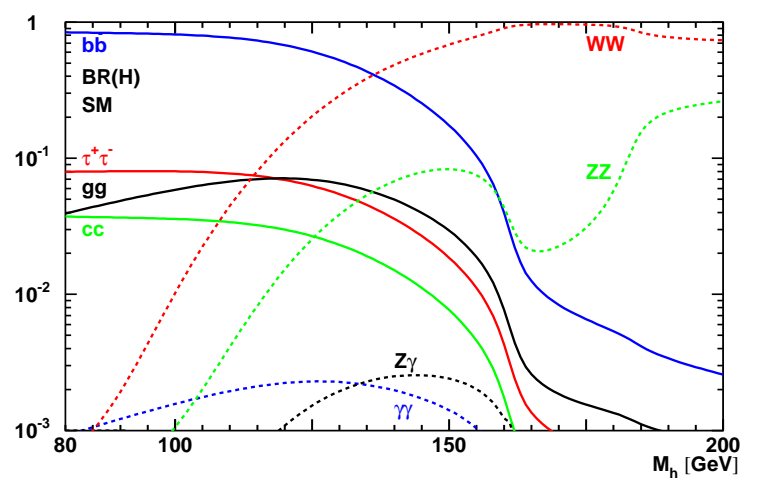

Figure 4: The branching ratios in the $\mathrm{SM}$ and $\mathrm{MCHM}_{4}$ as a function of $M_{H}$.

$\xi=0.5$ there is no sensitivity at all to a non-vanishing triple Higgs coupling because the triple Higgs coupling vanishes for this value.

However, these sensitivity areas represent only the ideal case where no background processes and detector effects are taken into account. To really answer the question whether the triple Higgs coupling can be measured an extensive analysis has to be done cf. also Ref. [23] for the SM. But we can give a rough estimate on the outcome of such an analysis. So for example for the $W^{+} W^{-} W^{+} W^{-} \rightarrow\left(j j l^{ \pm} v\right)\left(j j l^{ \pm} v\right)$ final state the dominating background processes are $\mathscr{O}\left(\alpha_{s} \alpha^{3}\right)$. They have at least two strong interaction vertices. This means that there is no Higgs boson in an 

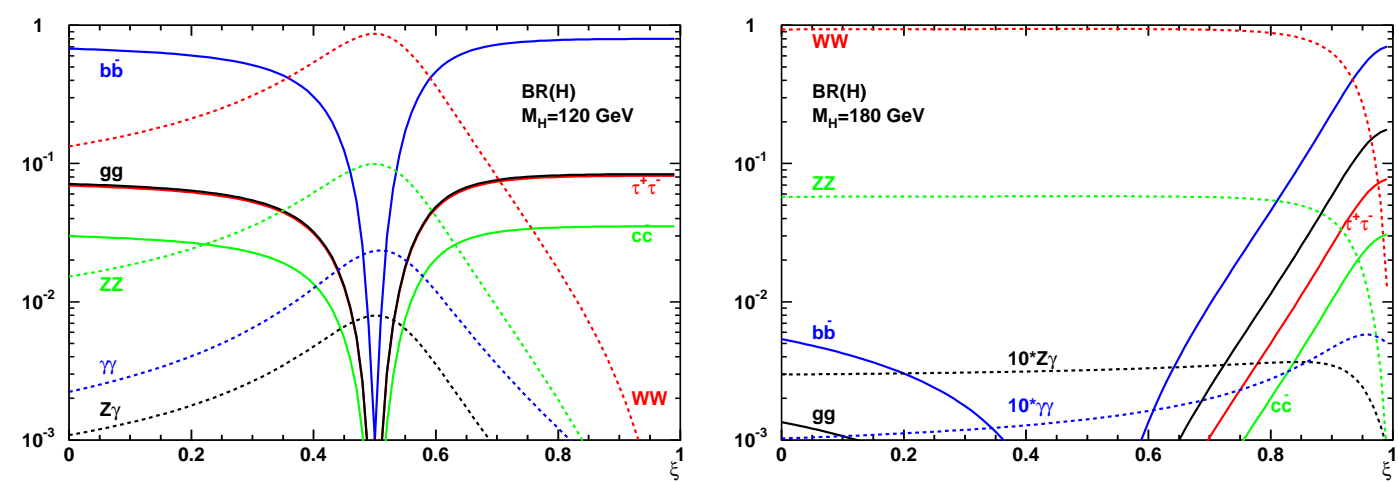

Figure 5: The branching ratios in $\mathrm{MCHM}_{5}$ as a function of $\xi$ for $M_{H}=120 \mathrm{GeV}$ (left) and $M_{H}=180 \mathrm{GeV}$ (right).
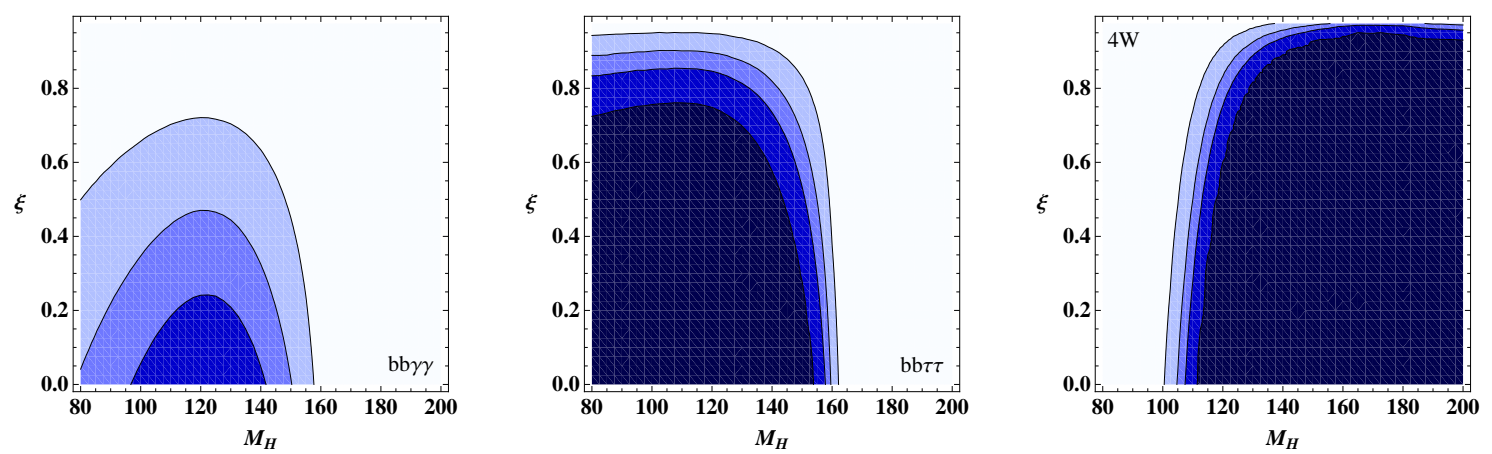

Figure 6: Areas in the $M_{H}-\xi$ plane with sensitivity to a non-vanishing $\lambda_{H H H}$ in $\mathrm{MCHM}_{4}$ for the gluon fusion process with subsequent decay. From dark blue to light blue the regions correspond to 5, 3,2, $1 \sigma$. The final states are from left to right $b \bar{b} \gamma \gamma, b \bar{b} \tau^{+} \tau^{-}$and $W^{+} W^{-} W^{+} W^{-}$. The assumed integrated luminosity is $\int \mathscr{L}=300 \mathrm{fb}^{-1}$.

intermediate state and therefore the dominating background processes are the same as for the SM. However, we saw that the signal cross section is enhanced compared to the SM. So in a first rough estimate the prospects of measuring the triple Higgs coupling look at least as good as in the SM or even better.

\section{Conclusion}

It has been shown that in the Minimal Composite Higgs models the cross sections for Higgs pair production via gluon fusion and vector boson fusion are enhanced compared to the SM whereas the cross sections of the Higgs-strahlung process is smaller than in the SM. The prospects of measuring a non-vanishing triple Higgs coupling in the gluon fusion process have been investigated. For the Higgs bosons decaying into $W^{+} W^{-} W^{+} W^{-}$and $b \bar{b} \tau^{+} \tau^{-}$there are large regions in the parameter space where the triple Higgs coupling might possibly be measured with an accuracy of $5 \sigma$. 

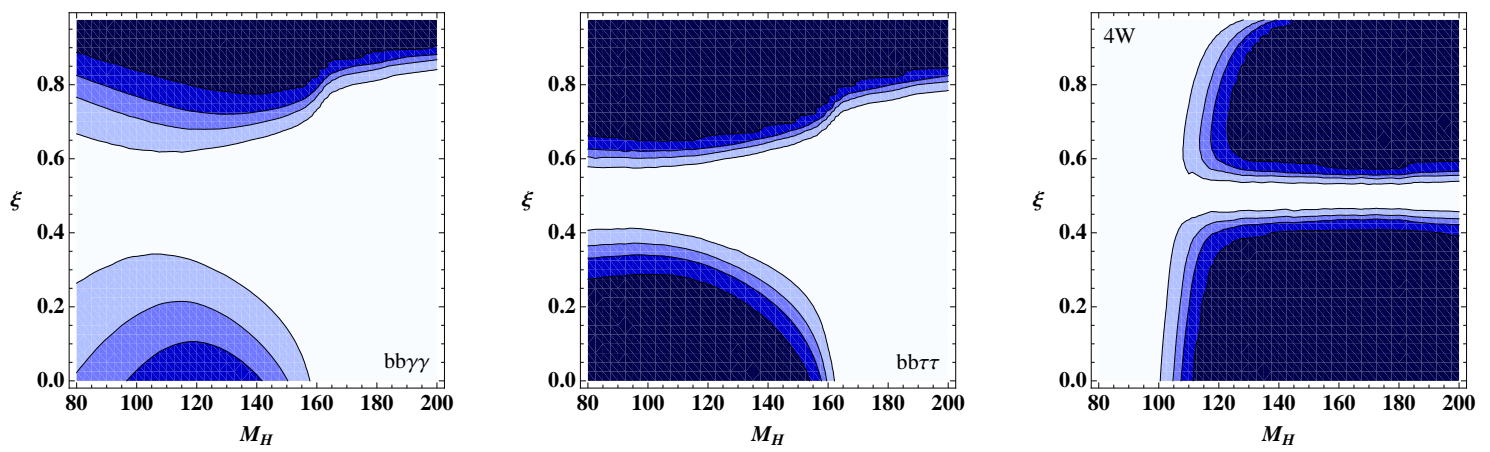

Figure 7: As in Fig. 6, but for $\mathrm{MCHM}_{5}$

\section{Acknowledgements}

R.G. would like to thank the organizers of "Corfu Summer Institute 2011 School and Workshops on Elementary Particle Physics and Gravity" for the possibility to give a talk and an inspiring atmosphere during the summer school. This research was supported in part by the Deutsche Forschungsgemeinschaft via the Sonderforschungsbereich/Transregio SFB/TR-9 Computational Particle Physics.

\section{References}

[1] J. Goldstone, A. Salam and S. Weinberg, Phys. Rev. 127 (1962) 965; S. Weinberg, Phys. Rev. Lett. 19 (1967) 1264; S.L. Glashow, S. Weinberg, Phys. Rev. Lett. 20 (1968) 224; A. Salam, Proceedings Of The Nobel Symposium, Stockholm 1968, ed. N. Svartholm.

[2] P.W. Higgs, Phys. Lett. 12 (1964) 132; and Phys. Rev. 145 (1966) 1156; F. Englert and R. Brout, Phys. Rev. Lett. 13 (1964) 321; P. W. Higgs, Phys. Rev. Lett. 13 (1964) 508; G.S. Guralnik, C.R. Hagen and T.W. Kibble, Phys. Rev. Lett. 13 (1964) 585.

[3] For a review see: J.F. Gunion, H.E. Haber, G. Kane and S. Dawson, The Higgs Hunters Guide, Addison-Wesley, 1990; M. Gomez-Bock, M. Mondragon, M. Mühlleitner, R. Noriega-Papaqui, I. Pedraza, M. Spira and P. M. Zerwas, J. Phys. Conf. Ser. 18 (2005) 74 [arXiv:hep-ph/0509077]; M. Gomez-Bock, M. Mondragon, M. Mühlleitner, M. Spira and P. M. Zerwas, arXiv:0712.2419 [hep-ph]; A. Djouadi, Phys. Rept. 457 (2008) 1 [hep-ph/0503172].

[4] D.V. Volkov and V.P. Alkulov, Phys. Lett. B46 (1973) 109; J. Wess and B. Zumino, Nucl. Phys. B70 (1974) 39; H.P. Nilles, Phys. Rep. 110 (1984) 1; H.E. Haber, G.L. Kane, Phys. Rep. 117 (1985) 75; M.F. Sohnius, Phys. Rep. 128 (1985) 39.

[5] D. B. Kaplan and H. Georgi, Phys. Lett. B 136 (1984) 183; S. Dimopoulos and J. Preskill, Nucl. Phys. B 199, 206 (1982); T. Banks, Nucl. Phys. B 243, 125 (1984); D. B. Kaplan, H. Georgi and S. Dimopoulos, Phys. Lett. B 136, 187 (1984); H. Georgi, D. B. Kaplan and P. Galison, Phys. Lett. B 143, 152 (1984); H. Georgi and D. B. Kaplan, Phys. Lett. B 145, 216 (1984); M. J. Dugan, H. Georgi and D. B. Kaplan, Nucl. Phys. B 254, 299 (1985).

[6] R. Grober, M. Muhlleitner, JHEP 1106, 020 (2011), [arXiv:1012.1562[hep-ph]].

[7] K. Agashe, R. Contino and A. Pomarol, Nucl. Phys. B 719 (2005) 165 [hep-ph/0412089].

[8] R. Contino, L. Da Rold and A. Pomarol, Phys. Rev. D 75 (2007) 055014 [hep-ph/0612048]. 
[9] G. F. Giudice, C. Grojean, A. Pomarol and R. Rattazzi, JHEP 0706 (2007) 045 [hep-ph/0703164].

[10] J. R. Espinosa, C. Grojean and M. Mühlleitner, JHEP 1005 (2010) 065 [arXiv:1003.3251 [hep-ph]].

[11] ATLAS Collaboration, ATLAS-CONF-2011-163; CMS Collaboration, CMS-PAS-HIG-11-032.

[12] J. R. Espinosa, C. Grojean and M. Muhlleitner, arXiv:1202.1286 [hep-ph]; J. R. Espinosa, C. Grojean, M. Muhlleitner and M. Trott, arXiv:1202.3697 [hep-ph]; A. Azatov, R. Contino and J. Galloway, arXiv:1202.3415 [hep-ph].

[13] T. Plehn, M. Rauch, Phys. Rev. D 72 (2005) 053008; A. Djouadi, W. Kilian, M. Muhlleitner and P. M. Zerwas, Eur. Phys. J. C 10 (1999) 45 [hep-ph/9904287].

[14] M. M. Muhlleitner, hep-ph/0008127.

[15] CLIC Physics working group, [hep-ph/0412251]; Eur. Phys. J. C 10 (1999) 27 [hep-ph/9903229].

[16] E. W. N. Glover and J. J. van der Bij, Nucl. Phys. B 309 (1988) 282.

[17] A. Dobrovolskaya and V. Novikov, Z. Phys. C 52 (1991) 427; D. A. Dicus, K. J. Kallianpur and S. S. D. Willenbrock, Phys. Lett. B 200 (1988) 187; A. Abbasabadi, W. W. Repko, D. A. Dicus and R. Vega, Phys. Rev. D 38 (1988) 2770; Phys. Lett. B 213 (1988) 386.

[18] V. D. Barger, T. Han and R. J. N. Phillips, Phys. Rev. D 38 (1988) 2766.

[19] S. Dawson, S. Dittmaier and M. Spira, Phys. Rev. D 58 (1998) 115012 [hep-ph/9805244].

[20] R. Contino, C. Grojean, M. Moretti, F. Piccinini and R. Rattazzi, JHEP 1005 (2010) 089, [arXiv:1002.1011 [hep-ph]].

[21] R. Contino, D. Marzocca, D. Pappadopulo and R. Rattazzi, JHEP 1110 (2011) 081, [arXiv:1109.1570 [hep-ph]].

[22] S. Bock, R. Lafaye, T. Plehn, M. Rauch, D. Zerwas and P. M. Zerwas, Phys. Lett. B 694 (2010) 44 [arXiv:1007.2645 [hep-ph]].

[23] U. Baur, T. Plehn and D. L. Rainwater, Phys. Rev. Lett. 89 (2002) 151801 [arXiv:hep-ph/0206024];

U. Baur, T. Plehn and D. L. Rainwater, Phys. Rev. D 67 (2003) 033003 [arXiv:hep-ph/0211224];

U. Baur, T. Plehn and D. L. Rainwater, Phys. Rev. D 68 (2003) 033001 [arXiv:hep-ph/0304015];

U. Baur, T. Plehn and D. L. Rainwater, Phys. Rev. D 69 (2004) 053004 [arXiv:hep-ph/0310056]. 